Pacific Journal of Mathematic 


\section{SOME PROPERTIES OF THE NASH BLOWING-UP}

\section{A. NoBILE}

Intuitively, in the Nash blowing-up process each singular point of an algebraic (or analytic) variety is replaced by the limiting positions of tangent spaces (at non-singular points). The following properties of this process are shown: 1) It is, locally, a monoidal transform; 2) in characteristic zero, the process is trivial if and only if the variety is nonsingular. Examples show that this is not true in characteristic $p>0$; that, in general, the transform of a hypersurface is not locally a hypersurface; and that this process does not give, in general, minimal resolutions.

Introduction. In this paper, the term algebraic variety (over a field $k$ ) means reduced, separated algebraic scheme over $k$; the term analytic variety means reduced, separated analytic space over $\mathbf{C}$, the complex numbers. Let $k$ be an algebraically closed field (resp. $k=\mathbf{C}$ ), $X$ a reduced closed subscheme of a Zariski open $U \subset \mathbf{A}^{n}$ (resp. a reduced closed complex subspace of an open $U \subset \mathbf{C}^{n}$ ) of pure dimension $r$, defined by $\left\{f_{1}, \cdots, f_{m}\right\} \subset \Gamma\left(U, \mathcal{O}_{U}\right)$. By the Nash blowing-up of $X$ we mean the pair $\left(X^{*}, p\right)$ obtained by the following process. Let $S(X)$ be the set of singular points of $X, X_{0}$ its complement in $X, \eta: X_{0} \rightarrow X \times G_{r}^{n}$ $\left(G_{r}^{n}\right.$ is the grassmanian of $r$-planes in $n$-space) the morphism determined by $\eta(x)=\left(x, T_{X, x}\right)$ for each closed point $x \in X_{0}$ (here $T_{X, x}$ is the tangent space of $X$ at $x$, which can be identified with an $r$-plane in $n$-space), $X^{*}$ the closure of $\eta\left(X_{0}\right)$ in $X \times G_{r}^{n}$ (resp. the closure in the metric topology), $p: X^{*} \rightarrow X$ induced by the first projection. In the complex case it is not obvious that $X^{*}$ is an analytic variety; see [7], Theorem 16.4 for a proof (or see Theorem 1 of this note).

It is possible to prove that $\left(X^{*}, p\right)$ is (up to unique $X$-isomorphism) independent of the immersion (as a locally closed subset) of $X$ in an affine space, hence the process globalizes.

Sketch of proof. Working (to simplify) in the algebraic case with closed points only, and calling $G_{r}(T)=\{r$-linear planes in $T\}$ for any vector space $T$, one verifies that $Z=\cup_{x \in X} x \times G_{r}\left(T_{X, x}\right)$ is a subvariety of $X \times G_{r}^{n}$, and $X^{*}$ is contained in $Z$. If $X^{\prime}$ is a locally closed in $\mathbf{A}^{m^{\prime}}$, we have (using notations as above, but with primes): $X^{\prime *} \subset X^{\prime} \times$ $G_{r}^{m^{\prime}}$. Assume $q: X \rightarrow X^{\prime}$ is an isomorphism. Then,

$$
(x, L) \rightarrow(q(x), d q(L)),
$$


for $(x, L) \in Z$, defines an isomorphism $Z \rightarrow Z^{\prime}$. This clearly induces an isomorphism $X^{*} \rightarrow X^{*}$, commuting with the projections.

A natural question, which apparently has not been seriously studied, is to determine the desingularization properties of this process. In this note we present some very basic results in this direction: (a) in characteristic zero, $p: X^{*} \rightarrow X$ is an isomorphism if and only if $X$ is nonsingular, (b) in positive characteristic, (a) is false. We also verify (which allows to show (b) in a very clear way) that, locally, a Nash blowing-up is a monoidal transform, with center a suitable ideal. The proof of (a) presented here is analytic, and uses results of $J$. Stutz on branched coverings (cf. [4] and [5]). It would be interesting to have an algebraic proof which probably would throw more light on the main question: if, in characteristic zero, this process desingularizes (cf. Remark 3).

We finish with some examples, which indicate other features of the process (see §3).

1. Monoidal transforms. In this section $k$ is, in the algebraic case, an algebraically closed field; in the analytic case $k=\mathbf{C}$. Our arguments hold in either case.

Recall that given a reduced subscheme $X$ of $\mathbf{A}_{k}^{n}$ (resp. a reduced subspace $X$ of an open $U$ in $\left.\mathbf{C}^{n}\right)$ and $\left\{g_{0}, \cdots, g_{s}\right\} \subset \Gamma\left(X, \mathscr{O}_{X}\right)$, the monoidal transform of $X$ with center $I=\left(g_{0}, \cdots, g_{s}\right)$ can be constructed by taking the closure (in $X \times \mathbf{P}^{s}$ ) of $\varphi(Y)$, where $Y=X \backslash V(I)$ $(V(I)=$ locus of $I)$ and $\varphi: Y \rightarrow X \times \mathbf{P}^{s}$ is defined by $\varphi(x)=$ $\left(x,\left(g_{0}(x), \cdots, g_{s}(x)\right)\right) \in X \times \mathbf{P}^{s}$, for any closed point $x$ (see [1], Remark 2).

REMARK 1. We shall use the following notations:

(1) We have two closed embeddings of $G_{r}^{n}$ in $\mathbf{P}^{N}, N=\left(\begin{array}{l}n \\ r\end{array}\right)-1$ :

(i) the map $\Lambda$, which sends the point corresponding to the $r$-plane $L$, of parametric equations $x_{i}=\sum_{d=1}^{r} b_{d}^{i} t_{d}, i=1, \cdots, n$, to the point of $\mathbf{P}^{N}$ of homogeneous coordinates $\left(\Delta_{i_{1} \cdots i_{r}}\right), 1 \leqq i_{1}<\cdots<i_{r} \leqq n$, where $\Delta_{i_{1} \cdots i_{r}}$ is the $r \times r$ subdeterminant of $\left\|b_{d}^{i}\right\|$ formed by the columns $i_{1}, \cdots, i_{r}$.

(ii) the map $\psi$, which sends the point corresponding to the $r$-plane $L$, defined by the equations $\sum_{j=1}^{n} a_{j}^{i} x_{j}=0, i=1, \cdots, n-r$, to the point of $\mathbf{P}^{N}$ of homogeneous coordinates $\left(\Delta_{j_{1} \ldots j_{n-r}}\right)$, where $\Delta_{j_{1} \ldots j_{n-r}}$ is the $(n-r) \times$ $(n-r)$ subdeterminant of $\left\|a_{j}^{i}\right\|$ defined by the columns $j_{1}, \cdots, j_{n-r}$. In the terminology of [2], Ch. VII, $\Lambda$ corresponds to the use of Grassman coordinates and $\psi$ to the dual Grassman coordinates; $\mathrm{cf}$. Theorem I, p. 294 of [2] for their relations. 
(2) Let $X, \eta$ be as in the introduction. We shall write

$$
\begin{aligned}
& \psi_{0}=(\mathrm{id} \times \psi) \eta: X_{0} \rightarrow X \times \mathbf{P}^{N} \\
& \Lambda_{0}=(\mathrm{id} \times \Lambda) \eta: X_{0} \rightarrow X \times \mathbf{P}^{N} .
\end{aligned}
$$

Clearly, there are natural isomorphisms:

$$
X^{*} \approx \operatorname{cl}\left(\psi_{0}\left(X_{0}\right)\right) \approx \operatorname{cl}\left(\Lambda_{0}\left(X_{0}\right)\right),
$$

where cl denotes closure in the corresponding ambient space.

(3) Given integers $n \geqq r>0, m \geqq n-r$, and an $(m \times n)$-matrix $\left\|a_{i j}\right\|=A$, let $S$ (resp. $S^{\prime}$ ) denote the set of increasing sequences of $(n-r)$-positive integers less than $m+1$ (resp. $n+1)$; if $\alpha=$ $\left(i_{1}, \cdots, i_{n-r}\right) \in S, \beta=\left(j_{1}, \cdots, j_{n-r}\right) \in S^{\prime}$, then $\Delta_{\alpha \beta}$ is the subdeterminant of $A$ obtained by considering the rows $i_{1}, \cdots, i_{n-r}$ and the columns $j_{1}, \cdots, j_{n-r}$.

Theorem 1. A Nash blowing-up is locally a monoidal tranform (with center a suitable ideal).

Proof. We may assume $X$ is affine (resp. an analytic set in $\left.U \subset \mathbf{C}^{n}\right)$; write $X$ as union of its irreducible components, $X=$ $X_{1} \cup \cdots \cup X_{d}$ (in the analytic case, shrink $U$ if necessary). Using the notations of the introduction, let $M=\left\|\partial f_{i} / \partial x_{j}\right\|, i=1, \cdots, m, j=$ $1, \cdots, n$. Clearly, for each $i=1, \cdots, d$, there is $\left(\alpha_{i}, \beta_{i}\right) \in S \times S^{\prime}$ such that $\Delta_{\alpha_{i} \beta_{i}}$ does not vanish on $X_{i}$; hence $W_{i}^{\prime}=X_{i} \backslash V\left(\Delta_{\alpha_{i} \beta_{i}}\right)$ is a nonempty open of $X_{i}$. For each $i=1, \cdots, d$, fix $h_{i} \in \Gamma\left(X, \mathscr{O}_{X}\right)$ such that $h_{i}=0$ on $\bigcup_{j \neq i} X_{j}, h_{i} \neq 0$ on $X_{i}$. Consider the ideal $I$ generated by $\left\{g_{\beta}\right\}, \beta \in S^{\prime}$, where $g_{\beta}=\sum_{i=1}^{d} h_{i} \Delta_{\alpha i \beta}$. We claim that the monoidal transform with center $I$ agrees with the Nash blowing-up of $X$.

In fact, first note that $V(I) \supset S(X)$. Call $W=X \backslash V(I)$, then (since all points in $X_{0} \backslash W$ are non-singular) $X^{*} \approx$ closure of $\psi_{0}(W)$ in $\mathbf{P}^{N}$. Hence, to show our contention we must check that the maps $W \rightarrow \mathbf{P}^{N}$ given by $p_{2} \psi_{0}=\psi_{1}$ ( $p_{2}$ is the second projection) and by $\left\{g_{\beta}\right\}$ agree. It is enough to check this at an arbitrary $z \in W_{i}=W_{i}^{\prime} \backslash V\left(h_{i}\right)$ $(i=1, \cdots, d)$. But for $z \in W_{i}$, as points of $\mathbf{P}^{N}$,

$$
\left(g_{\beta}(z)\right)=\left(\sum_{j=1}^{d} h_{j}(z) \Delta_{\alpha_{i} \beta}(z)\right)=\left(h_{i}(z) \Delta_{\alpha, \beta}(z)\right)=\left(\Delta_{\alpha i \beta}(z)\right)=\psi\left(L^{\prime}\right),
$$

where $L^{\prime}$ is the point of $G_{r}^{n}$ corresponding to the $r$-plane $L$, defined by $\left\{\Sigma\left(\partial f_{k_{l}}(z) / \partial x_{j}\right) x_{j}=0\right\},\left(k_{l}\right)=\alpha_{i}$, which is the tangent space to $X$ at $z$ (since $\Delta_{\alpha_{i} \beta_{i}} \neq 0$ ). As clearly $\psi\left(L^{\prime}\right)=\psi_{1}(z)$, the assertion is proved. 
REMARK 2. If $X$ (of dimension $r$ ) is defined by $n-r$ equations, then the proof is simpler. In fact, we may take $I$ to be the Jacobian ideal, formed by the $(n-r) \times(n-r)$ minors of $\left\|\partial f_{i} / \partial x_{j}\right\|, \quad i=$ $1, \cdots, n-r, j=1, \cdots, n$. In general this is not true, as the example of two planes in $\mathbf{A}^{4}$, meeting at one point, shows.

In this example also it can be seen that, in general, the support of the ideal of Theorem 1 is not the singular locus of $X$.

EXAMPLE 1. Let $\operatorname{ch}(k)=2$, consider the plane curve $y^{2}+x^{3}=$ 0. By Remark 1, its Nash blowing-up is the monoidal transform with center $I=\left(2 y, 3 x^{2}\right)=\left(x^{2}\right)$. This is a principal ideal, hence $p: X^{*} \rightarrow X$ is an isomorphism.

EXAMPLE 2. If $\operatorname{ch}(k)=q>2$, the Nash blowing-up of the plane curve $y^{2}-x^{q}=0$ is trivial. The verification is as in Example 1.

\section{Proof of the main theorem.}

THEOREM 2. Let $k$ be an algebraically closed field of characteristic zero (resp. $k=\mathrm{C}), X$ a pure $r$-dimensional algebraic (resp. analytic) variety over $k,\left(X^{*}, p\right)$ the Nash blowing-up of $X$. Then, $p$ is an isomorphism if and only if $X$ is nonsingular.

Proof. By descent theory we may assume, in the algebraic case, that $k=$ C. Moreover, it is clear (e.g., from Theorem 1) that $\left(X^{h}\right)^{*}=$ $\left(X^{*}\right)^{h}$ where $X^{h}$ denotes the analytic variety associated to an algebraic variety $X$. Hence, it suffices to prove the theorem in the analytic case.

One implication is obvious. Let us show that if $X$ has singularities the morphism $p$ is not an isomorphism. Let $S=S(X)$ be the singular set of $X$. We distinguish two cases.

Case a. $S$ has a component of codimension 1 .

Let $W$ be a component of codimension 1 . We claim that there is a point $x_{0} \in W$, such that $X$ can be embedded, locally about $x_{0}$, in a polydisk $U \subset \mathbf{C}^{n}$, in such a way that (with $x_{1}, \cdots, x_{n}$ coordinates in $\mathbf{C}^{n}$, and writing, to simplify, $X \subset U, W \subset U$, etc.):

(i) $x_{0}$ corresponds to the origin

(ii) $W$ is defined by $x_{r}=\cdots=x_{n}=0$.

(iii) Let $X_{1}, \cdots, X_{m}$ be the irreducible components of $X$. Then, there are analytic functions $f_{i j}\left(x_{1}, \cdots, x_{r}\right)$, defined on

$$
D=\left\{x \in \mathbf{C}^{r} /\left(x_{1}, \cdots, x_{r}, 0, \cdots, 0\right) \in U\right\}
$$


such that

$$
x \rightarrow\left(x_{1}, \cdots, x_{r-1}, x_{r}^{s_{i}}, f_{r+1, j}(x), \cdots, f_{n, j}(x)\right)
$$

defines a homeomorphism $D \rightarrow X_{j}, j=1, \cdots, m$.

(iv) The integer $s_{j}$ of (iii) is the multiplicity of $X_{j}$ at any $x \in W$, and

$$
f_{i j}=\sum_{k=s_{1}}^{\infty} a_{i j}^{(k)}\left(x_{1}, \cdots, x_{r-1}\right) x_{r}^{k}
$$

This is a consequence of the following results. Let $W_{0}=$ $\left\{x \in W: S\right.$ is nonsingular at $x, \operatorname{dim} C_{4}(X, x)=r, \operatorname{dim} C_{5}(X, x)=r+$ 1). Hence, $C_{i}(X, x), i=4,5$, are the indicated Whitney tangent cones to $X$ at $x$ (see [6], $\S 3$ for the definitions). In [4], Proposition 3.6, it is proved that $W_{0}$ is a dense open set in $W$. Propositions 2.5 of [5] and $4.2,4.6$ of [4] imply that for any $x_{0} \in W_{0}$, there is a local embedding of the type described above.

From now on, we shall assume that $X$ is contained in such an open $U \subset \mathbf{C}^{n}$. (The result that we are proving is clearly local on $X$.)

Note that now, keeping the notations of Remark 1, the map $\Lambda_{0}: X_{0} \rightarrow X \times \mathbf{P}^{N}$ can be described as follows: calling $\varphi_{i j}=x_{i}, 1 \leqq i \leqq$ $r-1, \varphi_{r j}=x_{r}^{s_{i}}, \varphi_{r+k, j}=f_{r+k, j}, k=1, \cdots, n-r$, then, for $j=1, \cdots, m$ :

$$
\Lambda_{0}:\left(\varphi_{i j}(x)\right)_{i=1, \cdots, n} \rightarrow\left(\left(\varphi_{i j}(x)\right)_{1 \leqq i \leqq n},\left(\Delta_{i_{1}}^{(j)}, \cdots, i_{r}\right)\right) \in X \times \mathbf{P}^{N},
$$

where $0<i_{1}<i_{2}<\cdots<i_{r} \leqq n$, and $\Delta_{i_{1}}^{(j)}, \cdots, i_{r}$ is the subdeterminant of $\left\|\partial \varphi_{i j} / \partial x_{k}\right\|, i=1, \cdots, n, k=1, \cdots, r$ formed of the rows $i_{1}, \cdots, i_{r}$. Note that $\Delta_{1,2, \cdots, r}^{(j)}=s_{j} x_{r}^{s_{r}-1}$.

We may assume $p: X^{*} \rightarrow X$ to be bijective (otherwise, the theorem is trivial). Then, if $A=\left\{x \in \mathbf{P}^{N} / z_{1, \cdots, r} \neq 0\right\}$, by using condition (iv) of the parametrization we see that $\operatorname{cl}\left(\Lambda_{0}\left(X_{0}\right)\right) \subset U \times A$. Let us identify, by using id $\times \Lambda$, the varieties $X^{*}$ and $\operatorname{cl}\left(\Lambda_{0}\left(X_{0}\right)\right)$. Then, the irreducible components $X_{j}^{*}$ of $X^{*} \subset U \times A$ are parametrized by:

$$
\left(\varphi_{i j}(x), \cdots, \varphi_{n j}(x),\left(s_{j}^{-1} x_{r}^{-s_{j}+1} \Delta_{i 1}^{(j)}, \cdots, i_{r}\right)\right),
$$

$1 \leqq i_{1}<\cdots<i_{r} \leqq n$ (except $\left.(1,2, \cdots, r)\right), j=1, \cdots, m$. By condition (iv), $\left(s_{j} x_{r}^{s_{r}-1}\right)^{-1} \Delta_{i_{1}}^{(j)}, \cdots, i_{r}$ are analytic functions.

Now there are two possibilities: (1) A component of $X$ is singular at $x_{0}$; (2) All components of $X$ are nonsingular at $x_{0}$.

To show that $p$ is not an isomorphism, it clearly suffices to show that if $X^{(q)}$ is the $q$ th iterated blowing-up of $X$ (i.e., $X^{(0)}=X, X^{(1)}=$ $\left.X^{*}, \cdots, X^{(q)}=\left(X^{q-1}\right)^{*}\right)$, then the induced canonical morphism 
$p_{q}: X^{(q)} \rightarrow X$ is not an isomorphism. We shall see that in either situation (1) or (2) above, this is the case.

Consider (1) first; let $X_{j}(j=1, \cdots, M \leqq m)$ be the components of $X$ which are singular at $x_{0}$. After changing coordinates (if necessary), we may assume that we have a parametrization of the components of $X$ satisfying (i) to (iv), and also:

$$
f_{r+1,1}=\sum_{k=d}^{\infty} a_{r+1,1}^{(k)}\left(x_{1}, \cdots, x_{r-1}\right) x_{r}^{k}
$$

where $a_{r+1,1}^{(d)} \not \equiv 0$, and $d$ is not a multiple of $s=s_{1}$. Write $(i)=$ $\left(i_{1}, \cdots, i_{r}\right)$, and, for $j=1, \cdots, m$,

$$
\left(s x_{r}^{s-1}\right)^{-1} \Delta_{(i)}^{(j)}=\psi_{(i)}^{(j)}=\sum b_{(i), k}^{(j)}\left(x_{1} \cdots x_{r-1}\right) x_{r}^{k} ;
$$

let $\psi_{(i)}^{(j)}=\psi_{(i)}^{(j)}-b_{(i), 0}^{(1)} . \quad$ Consider $X^{*} \subset U \times A$ (we maintain the identification of $X^{*}$ with cl $\Lambda_{0}\left(X_{0}\right)$ ). After an obvious change of coordinates we may assume that $p^{-1}(W)$ is defined, in $U \times A$, by $z_{i}=0, i \geqq r$, and the parametrization of $X_{j}^{*}=p^{-1}\left(X_{j}\right)$ induced by $(*)$ is:

$$
\left(x_{1}, \cdots, x_{r-1}, x_{r}^{s},\left(\psi_{(i)}^{(j)}\right)\right), \quad j=1, \cdots, m .
$$

If for some $j=1, \cdots, M$, there is an (i) such that $b_{(i), k}^{(j)} \not \equiv 0$ with $k<s_{j}$, then the multiplicity of $X_{j}^{*}$, at some point near $x_{0}$, is less than $s$, and hence $p$ is not an isomorphism. If not, then $(*)$ induces a parametrization of $X^{*} \subset(U \times A)$, satisfying (i) to (iv). We can repeat the process. We claim that after at most $\mu$ blowing-ups, with $\mu=[d / s]$, either $p_{\mu}$ is not bijective, or the multiplicity $s$ of $p_{\mu}^{-1}\left(X_{1}\right)$, at some point near $p_{\mu}^{-1}\left(x_{0}\right)$, drops. In fact, were $p_{0}=p, p_{1}, \cdots, p_{\mu}$ bijective, then one of the entries of the induced parametrization of $p_{\mu}^{-1}\left(X_{1}\right)$ is of the form

$$
\sum_{k=d}^{\infty} \gamma_{k, \mu} a_{r+1,1}^{(k)}\left(x_{1}, \cdots, x_{r-1}\right) x_{r}^{k-\mu s}
$$

where $\gamma_{k, \mu}=s^{-\mu} \prod_{v=1}^{\mu} k-(v-1) s$. Since $a_{q_{1}}^{(m)} \not \equiv 0$, and $(d, s)<n$, then $0<d-\mu s<s$ for $\mu=[d / s]$, and hence the multiplicity of such $X_{1}^{(\mu)}$, at some point near $p_{\mu}^{-1}\left(x_{0}\right)$, is less than $s$.

Consider the case (2). Since we assume that $p$ is bijective, then for all $x \in W, T_{X_{t}, x}=T_{X_{i}, x}, i=1, \cdots, m$. We also may assume, after a further change of coordinates, that, aside from (i) to (iv), we have: $X_{m}$ is the $r$-plane $x_{r+1}=\cdots=x_{r}=0$ (i.e., $f_{k, m}(x)=0, k=r+1, \cdots, n$ ). As before, we see that if the iterated blowing-ups

$$
p_{u}: X^{(u)} \rightarrow X^{(u-1)}, \quad 1 \leqq u \leqq q
$$


were bijective, then we can obtain a parametrization of the components of $X^{(q)}$, such that $X_{m}^{(q)}=p_{q}^{-1}\left(X_{m}\right)$ and $X_{1}^{(q)}=p_{q}^{-1}\left(X_{1}\right)$ are given, respectively, by $\left(x_{1}, \cdots, x_{r}, 0, \cdots, 0\right)$ and $\left(x_{1}, \cdots, x_{r}, \psi_{1}, \cdots, \psi_{L}\right)$ (for some integer $L)$ where, for some $i_{0}$,

$$
\psi_{i_{0}}=\sum_{k=d}^{\infty} \frac{k !}{(k-q) !} a_{r+1.1}^{(k)}\left(x_{1}, \cdots, x_{r-1}\right) x_{r}^{k-q} .
$$

If $a_{r+1,1}^{(k)}=0$ for $k<d$, and nonzero for $k=d$, then for $q=d-1, \psi_{i_{0}}$ has the form

$$
\psi_{i_{0}}=a\left(x_{1}, \cdots, x_{r-1}\right) x_{r}+\cdots, \quad a \neq 0 .
$$

Clearly, if $a\left(z_{1}, \cdots, z_{n}\right) \neq 0$, then $T_{X^{(a) . z}} \neq T_{X_{m}^{(a)}, z}$, and the next Nash blowing-up has at least two points lying over $z$. Hence, $p$ cannot be an isomorphism.

Case b. $S$ has codimension $>1$ at each of its points.

The only nontrivial case is the following: assume that for all $x \in S$, for all $\left\{x_{i}\right\} \rightarrow x, i=1,2, \cdots, x_{1}$ nonsingular, such that $\left\{T_{X, x_{i}}\right\}$ converges (in $G_{r}^{n}$ ), $\lim T_{X_{x} x_{i}}$ is a fixed space $T_{x}$ (otherwise, $p^{-1}(x)$ has more than one point). Assume this is the case. Pick any $x_{0} \in S$, and embed locally $X$ in a polydisk $U$ in $\mathbf{C}^{n}$ (as before, we just write $X \subset U, S \subset U$, etc.). Then, $C_{4}\left(X, x_{0}\right)$ has dimension $r$. In fact, this cone is the set of limit poisitions of lines, tangent to nonsingular points of $X$. By [7] (Part I, Preliminaries), the function $d: G_{r}^{n} \times \mathbf{P}^{1} \rightarrow \mathbf{R}$ where, for an $r$-plane $L \in G_{r}^{n}$ and a line $\ell \in \mathbf{P}^{1}, d(L, \ell)=$ distance between $L$ and $\ell$ (intuitively, the sine of the angle between $L$ and $\ell$ ) is continuous. From this, it follows that $C_{4}\left(X, x_{0}\right) \subset T_{x_{0}}=T$, hence $\operatorname{dim} C_{4}\left(X, x_{0}\right) \leqq r$. Since the inequality $\operatorname{dim} C_{4}\left(X, x_{0}\right) \geqq r$ always holds, we get $\operatorname{dim} C_{4}\left(X, x_{0}\right)=r$. By Proposition 2.6 of [4], this equality implies that, after shrinking $U$ if necessary, the projection $\pi$ on $T$, along a s(n-r)-plane transversal to $T$ satisfies: $B(\pi)=\{x \in X / \pi$ ramifies at $x\}=S$. Hence, $\operatorname{dim} B(\pi)<n-1$. This inequality implies, by the statement 1.8 of [4], that (after further shrinking of $U$, if necessary) all the irreducible components $X_{i}$ of $X$ are nonsingular, and $S(X)=$ $B(\pi)=\bigcup_{i \neq j}\left(X_{\imath} \cap X_{j}\right)$. Thus, there is more than a component at $x_{0}$ (since $x_{0}$ is a singular point of $X$ ), and $T_{X_{t} x_{0}}=T$ for all $i$. By changing coordinates (if necessary), we may assume $X_{1}=T$, and by the implicit function theorem we may parametrize simultaneously the components $X_{1}$ :

$$
\left(x_{1}, \cdots, x_{r}, \varphi_{r+1, j}\left(x^{\prime}\right), \cdots, \varphi_{n, j}\left(x^{\prime}\right)\right), \quad x^{\prime}=\left(x_{1}, \cdots, x_{r}\right) .
$$


Exactly as in part (2) of case (a), we see that after a finite number of Nash blowing-ups, the components of $X$ get separated, and hence $p$ cannot be an isomorphism.

The proof of the theorem is complete.

We have the following corollary, which seems to be well known:

COROLlARY 1. If $C$ is an algebraic curve, defined over an algebraically closed field a characteristic zero (resp. an analytic curve), a finite sequence of Nash blowing-ups desingularizes the curve.

Proof. In general, the Nash blowing-up $p: C^{*} \rightarrow C$ is a finite birrational (resp. bimeromorphic) morphism. Then it is clear that after a finite number of Nash blowing-ups, we reach the normalization. Since this is nonsingular, the result follows.

As we saw, this is false in positive characteristic.

REMARK 3. In [7], J. Lipman proves, in a purely algebraic way, that for an algebraic variety $X$, the monoidal transform with center the sheaf of Jacobian ideals is trivial if and only if $X$ is smooth. Since, by Remark 2, for complete intersections this transform agrees with the Nash blowing-up, it gives an algebraic proof of Theorem 2 in this case.

3. Some remarks and examples. In general, the Nash blowing-up of a hypersurface is not locally a hypersurface, as the following example shows.

ExAmple 3. Let $\operatorname{char}(k)=0$. Consider the plane curve $X$ of parametric equations:

$$
x=t^{4}, \quad y=\varphi(t)=t^{11}+t^{13} .
$$

Let $p: X^{*} \rightarrow X$ be the Nash blowing-up, $x_{0} \in X$ the origin. Then $p^{-1}\left(x_{0}\right)$ has only one point $x_{1}$, and a neighborhood $X_{1}$ of $x_{1}$ in $X^{*}$ is naturally contained in $\mathbf{A}^{2} \times \mathbf{A}^{1} \subset \mathbf{A}^{2} \times G_{1}^{2}$, and has a parametrization

$$
x=t^{4}, \quad y=t^{11}+t^{13}, \quad u=\frac{11}{4} t^{7}+\frac{13}{4} t
$$

(cf. proof of Theorem 2). We claim that the embedding dimension of $X_{1}$ at $x_{1}$ is 3 . In fact, if emb. $\operatorname{dim}_{x_{1}} X_{1}=2$, it would follow

$$
y=t^{11}+t^{13}=g\left(t^{4}, \frac{11}{4} t^{7}+\frac{13}{4} t^{9}\right)
$$

for some $g(x, u) \in k[[x, u]]$. An elementary computation shows that this is impossible. 
Even if it were true that the Nash blowing-up process desingularizes, in general one would not get "minimal" resolutions. Consider this example:

Example 4. Let $\operatorname{ch}(k) \neq 2$. Consider the surface $X$ (in $\mathbf{A}^{3}$ ) defined by $y^{2}-x^{2} z=0$. It is well known that the normalization $X^{\prime}$ of this surface is nonsingular. Moreover, this normalization can be obtained by applying the monoidal transform with center the $z$-axis. Thus, one can get a desingularization $\pi: X^{\prime} \rightarrow X$, with $\pi$ finite. However, the Nash blowing-up $p: X^{*} \rightarrow X$ is not finite, in fact $p^{-1}(0)$ is a projective line. But $X^{*}$ is nonsingular; in fact, using Remark 1 , and the fact that (for any ideal $I$ ), the monoidal transforms with center the ideal $I$ and $I^{2}$ coincide, it is easy to see that the Nash blowing-up of $X$ can be obtained by composing $\pi: X^{\prime} \rightarrow X$ and the quadratic transform of $X^{\prime}$ with center the point $\pi^{-1}(0)$.

\section{REFERENCES}

1. H. Hironaka, and M. Rossi, On the equivalence of imbeddings of exceptional complex spaces, Math. Annalen, 156 (1964), 313-333.

2. W. Hodge, and B. Pedoe, Methods of Algebraic Geometry, Vol. 1, Cambridge U. Press, 1947.

3. J. Lipman, On the Jacobian ideal of the module of differentials, Proc. Amer. Math. Soc., 21, (1969), 422-426.

4. J. Stutz, Analytic sets as branched coverings, Trans. Amer. Math. Soc., 166 (1972), 241-259.

5. - Equisingularity and equisaturation in codimension 1, Amer. J. Math., XCIV (1972), $1245-1268$.

6. H. Whitney, Local properties of analytic varieties, Differential and Combinatorial Topology, (Edited by S. Cairns), Princeton U. Press, 1965.

7. _ Tangents to an analytic variety, Ann. Math., 81 (1965), 496-549.

Received June 12, 1974 and in revised form November 1, 1974. 

D. E. Bennett, Strongly unicoherent continua ............................. 1

Walter R. Bloom, Sets of p-spectral synthesis ................................ 7

R. T. Bumby and D. E. Dobbs, Amitsur cohomology of quadratic extensions: Formulas and number-theoretic examples ................. 21

W. W. Comfort, Compactness-like properties for generalized weak topological sums

D. R. Dunninger and J. Locker, Monotone operators and nonlinear biharmonic boundary value problems ...

T. S. Erickson, W. S. Martindale, 3rd and J. M. Osborn, Prime nonassociative algebras

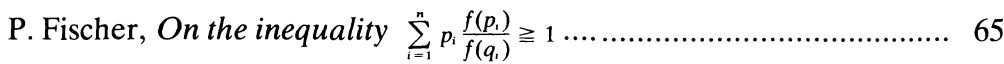

G. Fox and P. Morales, Compact subsets of a Tychonoff set ............... 75

R. Gilmer and J. F. Hoffmann, A characterization of Prüfer domains in terms of polynomials ......................................................... 81

L. C. Glaser, On tame Cantor sets in spheres having the same projection in each direction ......................................................... 87

Z. Goseki, On semigroups in which $X=X Y X=X Z X$ if and only if

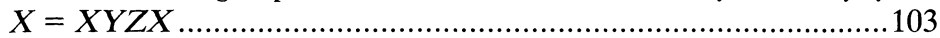

E. Grosswald, Rational valued series of exponentials and divisor

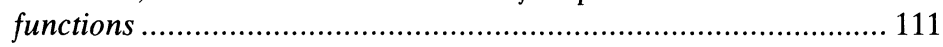

D. Handelman, Strongly semiprime rings ...................................... 115

J. N. Henry and D. C. Taylor, The $\bar{\beta}$ topology for $w^{*}$-algebras ............. 123

M. J. Hodel, Enumeration of weighted p-line arrays .......................... 141

S. K. Jain and S. Singh, Rings with quasiprojective left ideals .............. 169

S. Jeyaratnam, The diophantine equation $Y(Y+m)(Y+2 m) \times$

$$
(Y+3 m)=2 X(X+m)(X+2 m)(X+3 m) \ldots \ldots \ldots \ldots \ldots \ldots \ldots . . .183
$$

$\mathrm{R}$. Kane, On loop spaces without $p$ torsion .........................................189

Alvin J. Kay, Nonlinear integral equations and product integrals ..........203

A. S. Kechris, Countable ordinals and the analytic hierarchy, I ...........223

Ka-Sing Lau, A representation theorem for isometries of $C(X, E) \ldots \ldots . .229$

I. Madsen, On the action of the Dyer-Lashof algebra in $H_{*}(G)$..........235

R. C. Metzler, Positive linear functions, integration, and Choquet's theorem ........................................................................................ 277

A. Nobile, Some properties of the Nash blowing-up ............................297

G. E. Petersen and G. V. Welland, Plessner's theorem for Riesz conjugates 


\section{Pacific Journal of Mathematics}

\section{Vol. 60, No. $1 \quad$ September, 1975}

Donald Earl Bennett, Strongly unicoherent continua ................ 1

Walter Russell Bloom, Sets of p-spectral synthesis ................ 7

Richard Thomas Bumby and David Earl Dobbs, Amitsur cohomology of

quadratic extensions: formulas and number-theoretic examples .......

W. Wistar (William) Comfort, Compactness-like properties for generalized

weak topological sums ...................................

Dennis Robert Dunninger and John Stewart Locker, Monotone operators

and nonlinear biharmonic boundary value problems ..............

Theodore Erickson, Wallace Smith Martindale, III and J. Marshall Osborn,

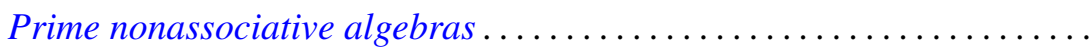

Pál Fischer, On the inequality $\sum_{i=0}^{n}\left[f\left(p_{i}\right) / f\left(q_{i}\right)\right] p_{i} \geq i \ldots \ldots \ldots \ldots \ldots$

Geoffrey Fox and Pedro Morales, Compact subsets of a Tychonoff set.......

Robert William Gilmer, Jr. and Joseph F. Hoffmann, A characterization of

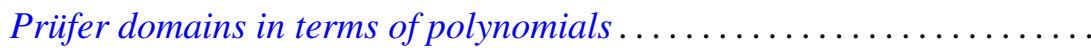

Leslie C. Glaser, On tame Cantor sets in spheres having the same projection

in each direction . ...................................

Zensiro Goseki, On semigroups in which $x=x y x=x z x$ if and only if

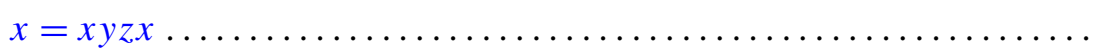

Emil Grosswald, Rational valued series of exponentials and divisor

functions.

David E. Handelman, Strongly semiprime rings

Jackson Neal Henry and Donald Curtis Taylor, The $\bar{\beta}$ topology for

$W^{*}$-algebras

Margaret Jones Hodel, Enumeration of weighted p-line arrays ...

Surender Kumar Jain and Surjeet Singh, Rings with quasi-projective left

ideals.

S. Jeyaratnam, The Diophantine equation

$$
Y(Y+m)(Y+2 m)(Y+3 m)=2 X(X+m)(X+2 m)(X+3 m) \ldots
$$

Richard Michael Kane, On loop spaces without $p$ torsion

Alvin John Kay, Nonlinear integral equations and product integrals ...

Alexander S. Kechris, Countable ordinals and the analytical hierarchy.

$I$.

Ka-Sing Lau, A representation theorem for isometries of $C(X, E)$

Ib Henning Madsen, On the action of the Dyer-Lashof algebra in $H_{*}(G)$

Richard C. Metzler, Positive linear functions, integration, and Choquet's

theorem.

Augusto Nobile, Some properties of the Nash blowing-up

Gerald E. Peterson and Grant Welland, Plessner's theorem for Riesz. 